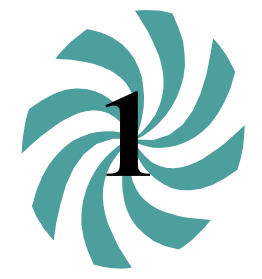

Tecnociencia, Vol. 23, $\mathrm{N}^{\circ} 1: 5-25$

Enero- Junio 2021

\title{
EI VALLE DE ANTÓN, PROVINCIA DE COCLÉ; COMO SITIO DE PASO Y OBSERVACIÓN DE AVES MIGRATORIAS EN PANAMÁ
}

\author{
Nelson Guevara A. (D) 1,2 y Nicole Samudio ${ }^{1,2}$
}

${ }^{1}$ Universidad de Panamá, Facultad de Ciencias Naturales, Exactas y Tecnología, Escuela de Biología. ${ }^{2}$ Grupo Biológico Biomundi; e-mail: bio.mundi18@gmail.com; nssr8888@gmail.com

\begin{abstract}
RESUMEN
Panamá siendo un puente entre ambos extremos del continente, cuenta con una gran abundancia y diversidad de aves migratorias las cuales visitan los diferentes puntos del país, a pesar de esto, aún se desconoce con certeza cuales son las zonas más visitadas por estas durante su proceso de migración. Entre octubre y noviembre de 2019, se realizaron observaciones en El Valle Antón, Provincia de Coclé, con el objetivo de determinar si por sus condiciones naturales, urbanísticas y altura, era un punto propicio de observación de estas especies las cuales realizan altos vuelos altitudinales. Por medio de la búsqueda generalizada se obtuvo un registro de 544 individuos, 32 especies, 9 familias y 3 órdenes, obteniendo el $22 \%$ de las aves migratorias registradas para el país; el orden Passeriformes fue el más diverso y abundante con 7 familias, 27 especies y 526 individuos. A pesar del avance urbanístico que presenta El Valle de Antón, este puede ser considerado como sitio de paso dentro de las rutas migratorias a seguir por las diferentes especies e individuos, sin embargo, el cuidado de estos espacios verdes, jardines y grandes bordes de bosque dentro o cerca del poblado en $\mathrm{El}$ Valle es de suma importancia para el aseguramiento de la visita anual de las especies neárticas.
\end{abstract}

\section{PALABRAS CLAVES}

Migración, Valle de Antón, Observación, diversidad de aves migratorias, avance urbanístico. 


\title{
EI VALLE DE ANTÓN, COClÉ PROVINCE; AS A SITE OF PASSAGE AND OBSERVATION OF MIGRATORY BIRDS IN PANAMA
}

\begin{abstract}
Panama being a bridge between both ends of the continent, it has a great abundance and diversity of migratory birds which visit the different parts of the country, despite this, it is still unknown with certainty which are the areas most visited by them during their process of migration. Between October and November 2019, observations were made in El Valle de Antón in the Province of Coclé, with the aim of determining whether, by its natural, urban and altitude conditions, it was a favorable point of observation for these species that perform high altitude flights. Through the generalized search, a record of 544 individuals, 32 species, 9 families and 3 orders were obtained, obtaining $22 \%$ of the migratory birds registered for the country; the order Passeriformes being the most diverse and abundant with 7 families, 27 species and 526 individuals. Despite the great urban development that El Valle de Antón presents, it can be considered as a transit point within the migratory routes to be followed by the different species and individuals, however, the care of these green spaces, gardens and large edges of forest in or near the El Valle village is of utmost importance to ensure the annual visit of Neartic species.
\end{abstract}

\section{KEYWORDS}

Migration, Valle de Antón, Observation, diversity of migratory birds, urban advance.

\section{INTRODUCCIÓN}

A lo largo de la historia el estudio y observación de aves, tanto por profesionales como por aficionados, es una de las actividades con mayor aumento en los últimos años (Eisermann, 2006), volviéndose uno de los grupos zoológicos que ha despertado una gran admiración y simpatía debido a su belleza y comportamiento (Méndez, 1969). El Istmo de Panamá posee una significativa diversidad de aves, 1009 especies en total (AUDUBON, 2019). De este total, la avifauna panameña cuenta con un total de 150 especies migratorias, provenientes de Norte América, específicamente Estados Unidos, Canadá y México (Angehr \& Dean, 2010). Cada año visitan los bosques, playas y espacios disponibles en busca de refugio, reposo y alimento, excepto zonas de reproducción, prácticamente todas las aves migratorias de larga distancia crían en los climas templados o árticos de Norteamérica (Ridgely \& Gwynne, 1976). 
El fenómeno de migración se ha vuelto uno de los más conspicuos y extraordinarios del continente, debido a la gran cantidad de especies e individuos que se desplazan siguiendo patrones de estacionalidad y orientación geográfica para establecer sitio de visitas anuales durante su ciclo de vida (Ocampo, 2010). Estos viajes anuales de las aves neárticas son realizados en dos épocas del año, otoño boreal y primavera (Ridgely y Gwynne, 1993).

\section{MATERIALES Y MÉTODOS}

El estudio se realizó en El Valle de Antón (8³6’07 N y 8007`15’`W), distrito de Antón, Provincia de Coclé, Republica de Panamá (Fig. 1). Cuenta con un clima tropical húmedo, con influencia del monzón (régimen de vientos), caracterizado por una precipitación promedio anual menor a $2250 \mathrm{~mm}$ con una humedad relativa del $60 \%$ concentrada en los cuatro meses más lluviosos de forma consecutiva y temperatura promedio de $18^{\circ} \mathrm{C}$ del mes más fresco, según la clasificación de Köpen (ETESA, 2007).

El Valle de Antón según la clasificación de Holdridge (1979), está ubicado entre las zonas de vida denominadas bosque muy húmedo tropical (Bht)/bosque pluvial premontano (Bpp). Se localiza en la ecorregión denominada bosque húmedo del lado Pacifico del Istmo, con una cobertura boscosa compuesta por su altura, en dos tipos de bosques a su alrededor: bosque maduro y bosque secundario maduro de tierras que se encuentran de 701 a 1,200 metros en el Pacifico, y tres tipos de vegetación según la clasificación de la UNESCO: bosque perennifolio ombrófilo tropical, latifoliado de tierras bajas-bastante intervenida; bosque perennifolio ombrófilo tropical, latifoliado submontano y sistema productivo con vegetación leñosa natural o espontanea significativa (ANAM, 2010).

El valle cuenta con un relieve de pendientes que pueden estar entre los $0^{\circ}$ a $3^{\circ}$ (poco inclinada) y entre $\operatorname{los} 16^{\circ}$ a $30^{\circ}$ (fuertemente inclinada). Las características físicas y de relieve indican que El Valle es de origen volcánico, compuesto por coladas de lavas, intercaladas con piroclastitas (cenizas, lapilis y bloques) de naturaleza riolítica, las cuales se pueden evidenciar por las constantes fracturas, hundimientos, zonas altas y bajas; y levantamientos observadas en el área (IGNTN, 1988). Los suelos están caracterizados por ser no arable, con limitaciones severas o arables, severas limitaciones en la selección de plantas en 
algunos puntos, clasificándolo como suelo de tipo VII, no volviendo la zona apta para la agricultura, pero si para la conservación de flora nativa (ANAM, 2010).
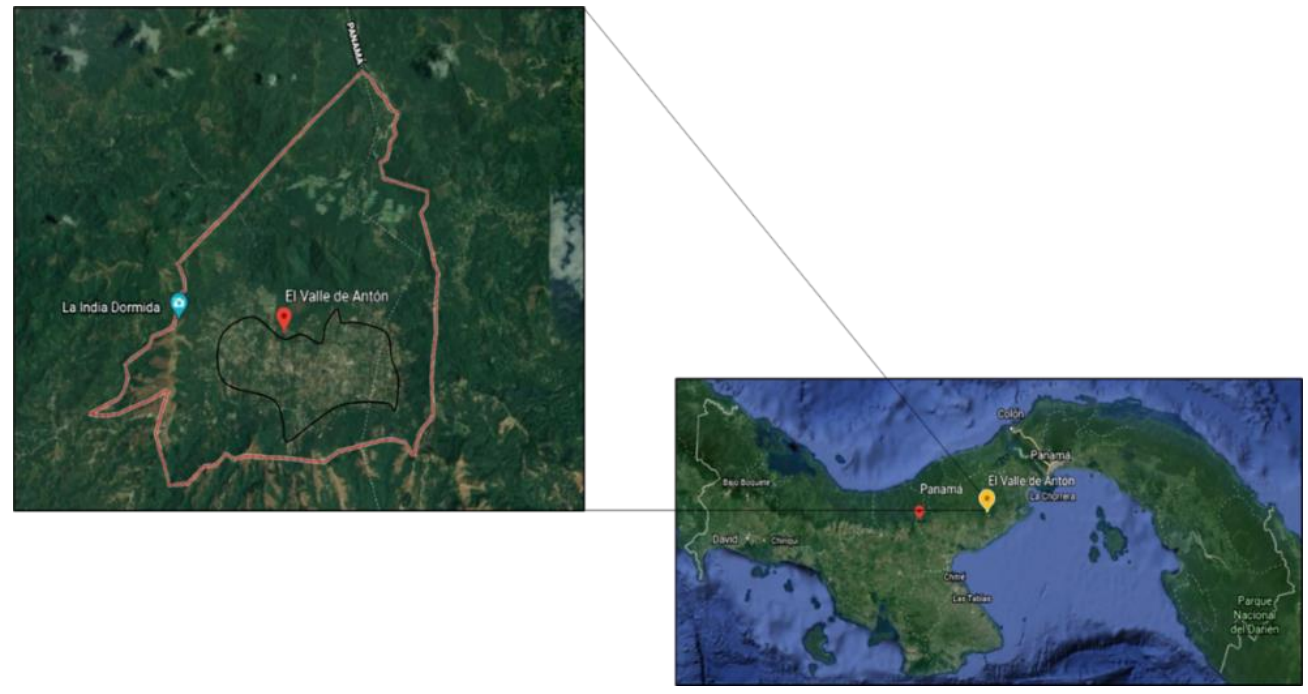

Fig. 1. Vista panorámica. El Valle de Antón (Google s.f.). [Localización de El Valle de Antón, Provincia de Coclé, Google Earth]. Recuperado el 13 de enero del 2020. (Rojo-Territorio de El Valle de Antón, negro-área principal de muestreo).

\section{TRABAJO EN CAMPO}

\section{Toma de datos}

El estudio se realizó entre los meses de octubre y noviembre del 2019. Con un total de 10 muestreos, divididos en 5 muestreos por mes. Para la identificación de las especies se utilizaron binoculares Nikon MONARCH M511 8X42 6.3 ${ }^{\circ}$ Waterproof, Cámara Profesional Canon Rebel T6 y las guías de aves de Ridgely \& Gwynne (1993) y Angehr \& Dean (2010). Para la clasificación taxonómica de las especies, se utilizó el arreglo de la Sociedad AUDUBON (2019). Finalmente, para la toma de datos se utilizó la técnica de búsqueda generaliza. Método apropiado para la compilación de información de un área determinada, principalmente cuando se dispone de poco tiempo (Ralph et al., 1996). El método será descrito a continuación: 


\section{Búsqueda Generalizada}

Se realizaron recorridos desde las 6:00. a las 18:00. horas. Se tomo como punto de inicio el lugar más céntrico del poblado del El Valle conocido como el mercado, de forma aleatoria se iniciaban los recorridos por el poblado hacia las zonas con mayor representación boscosa en el lugar, moviéndose al borde de esta. Los datos tomados al realizar las observaciones: especies, número de individuos, hora, acción realizada por el individuo (posada, alimentándose, movimiento \{individuos moviéndose entre la vegetación \} o en vuelo \{para las aves encontradas desplazándose a gran altura\}). Esto se realizó tomando en cuenta solamente las especies reconocidas como migratorias neárticas por el Laboratorio Ornitológico de Cornell-Cornell Lab of Ornithology de Ithaca, New York, Estados Unidos y La Sociedad AUDUBON de Panamá (AUDUBON, 2020).

\section{Análisis de datos}

Para el análisis matemático de los datos se utilizó el programa Microsoft Excel 2019, para la confección de la curva acumulativa de especie se utilizó el programa EstimateS 9 Windows. Se utilizaron los estimadores Chaol y Ace para estimar el porcentaje de riqueza de especie obtenidos en el lugar (Moreno, 2001).

\section{RESULTADOS Riqueza de especies}

En total, se registraron 544 individuos, comprendidos en 32 especies, 9 familias y 3 órdenes (Anexo 1). Siendo Petrochelidon pyrrhenota con el $22 \%$ de los registros (Fig. 2), la especie más representativa en cuanto números de individuo (125), seguido de Vireo flavoviridis $11 \%$ con 59 individuos (Fig. 3) y Vireo olivaceus $10 \%$ con 54 individuos (Fig. 4). 


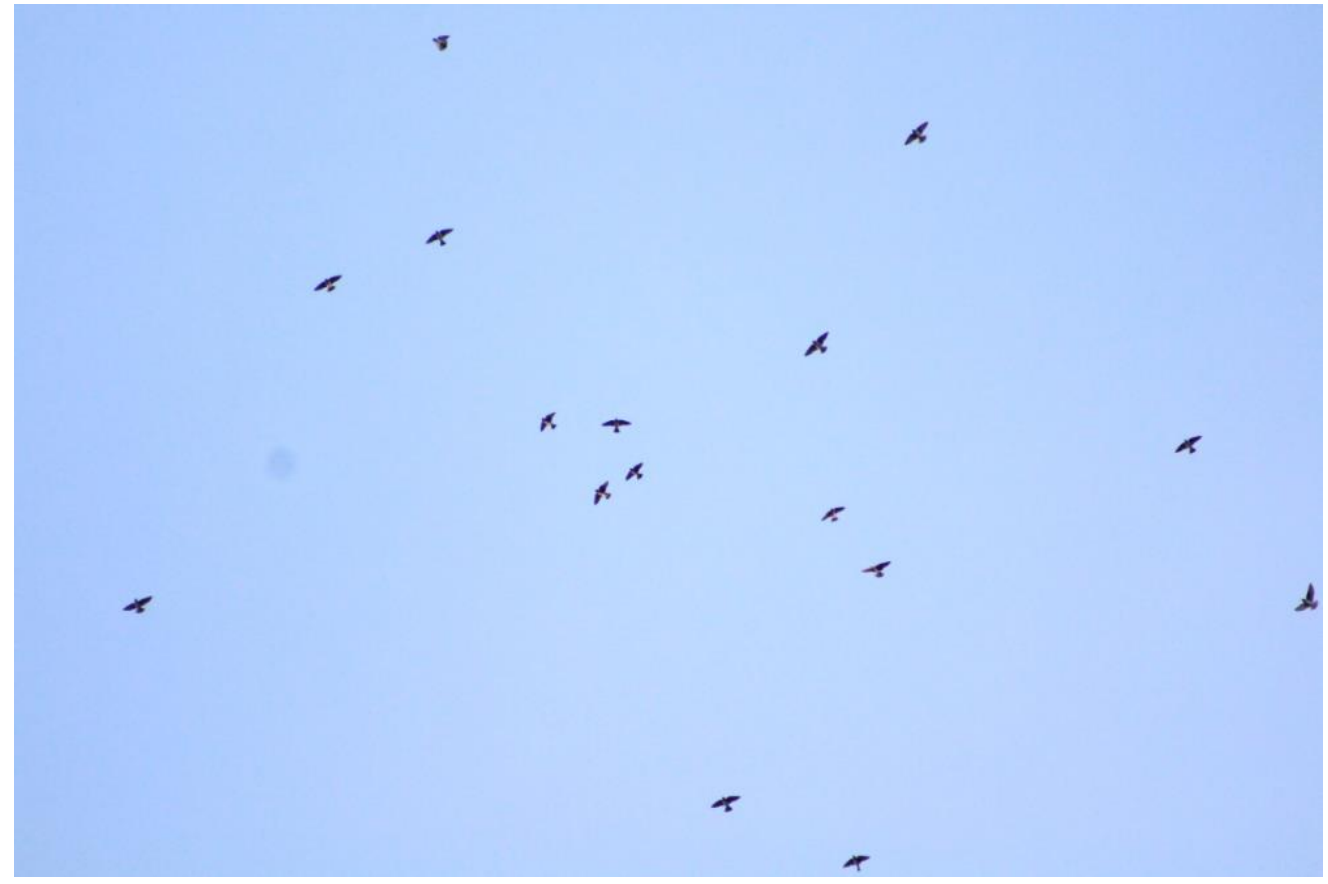

Fig. 2. Golondrina risquera (petrochelidon pyrrhonota). Grupo sobrevolando en El Valle de Antón.

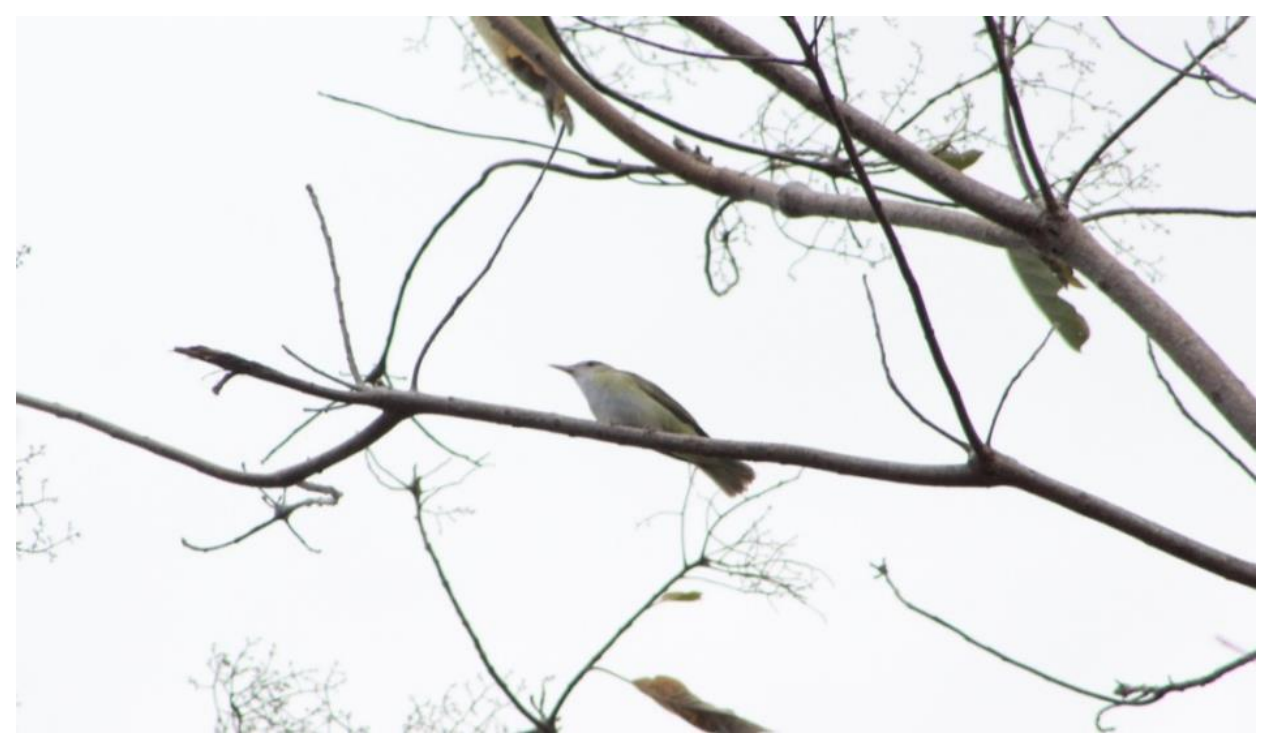

Fig. 3. Víreo verdiamarillo (Vireo flavoviridis). Individuo posado en una rama cerca a la vía principal en El Valle de Antón. 


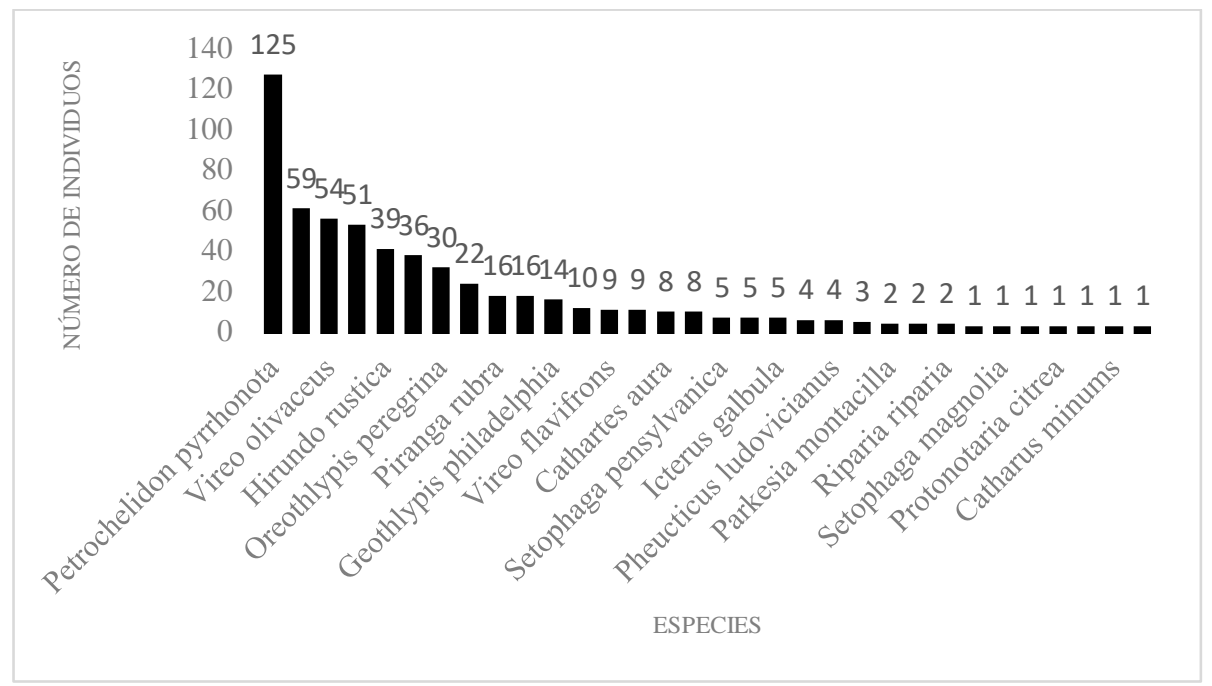

Fig. 4. Número de individuos registrados por especies.

En cuanto a las taxas, el orden Passeriformes fue el mayor representado con 7 familias (80\%), 27 especies (85\%) y 526 individuos (96\%). Seguido de Accipitriformes con una familia (10\%), 4 especies $(10 \%)$ y 19 individuos (3\%) y Cathertiformes con una familia (10\%), una especie (5\%) y 8 individuos (1\%) (Cuadro 1). En cuanto a las familias, la mayor representada fue Parulidae con 13 especies (40.62\%), 132 individuos (24.26\%), seguido de Hirundinidae con 3 especies (9.37\%), 162 individuos (29.77\%) y Vireonidae con 3 especies (9.37\%) y 122 individuos $(22.42 \%)$ (Cuadro 2)

Cuadro 1. Resultados obtenidos a nivel de Orden.

\begin{tabular}{lcccccc}
\hline Orden & Familia & \% & Especies & \% & Individuos & \% \\
\hline Cathartiformes & 1 & 10 & 1 & 3 & 8 & 1 \\
\hline Accipitriformes & 1 & 10 & 4 & 12 & 19 & 3 \\
\hline Passeriformes & 7 & 80 & 27 & 85 & 526 & 96 \\
\hline
\end{tabular}


Cuadro 2. Resultados obtenidos a nivel de Familia.

\begin{tabular}{ccccc}
\hline Familia & Especies & $\boldsymbol{\%}$ & Individuos & $\boldsymbol{\%}$ \\
\hline Cathartidae & 1 & 3.12 & 8 & 1.5 \\
\hline Accipitridae & 4 & 12.5 & 19 & 3.5 \\
\hline Tyrannidae & 2 & 6.25 & 4 & 0.73 \\
\hline Virenonidae & 3 & 9.37 & 122 & 22.42 \\
\hline Hirundinidae & 3 & 9.37 & 162 & 29.77 \\
\hline Turdidae & 2 & 6.25 & 52 & 10.00 \\
\hline Parulidae & 13 & 40.62 & 132 & 24.26 \\
\hline Cardinalidae & 3 & 9.37 & 36 & 7.00 \\
\hline Icteridae & 1 & 3.12 & 5 & 0.91 \\
\hline & & & &
\end{tabular}

\section{Esfuerzo}

Se realizaron 10 muestreos con un esfuerzo total de 240:00 horas. Obteniendo un número representativo de especies migratorias en El Valle de Antón. Según los estimadores Chao1 y Ace, se obtuvo aproximadamente el 80\% de especies migratorias (Fig. 5). 


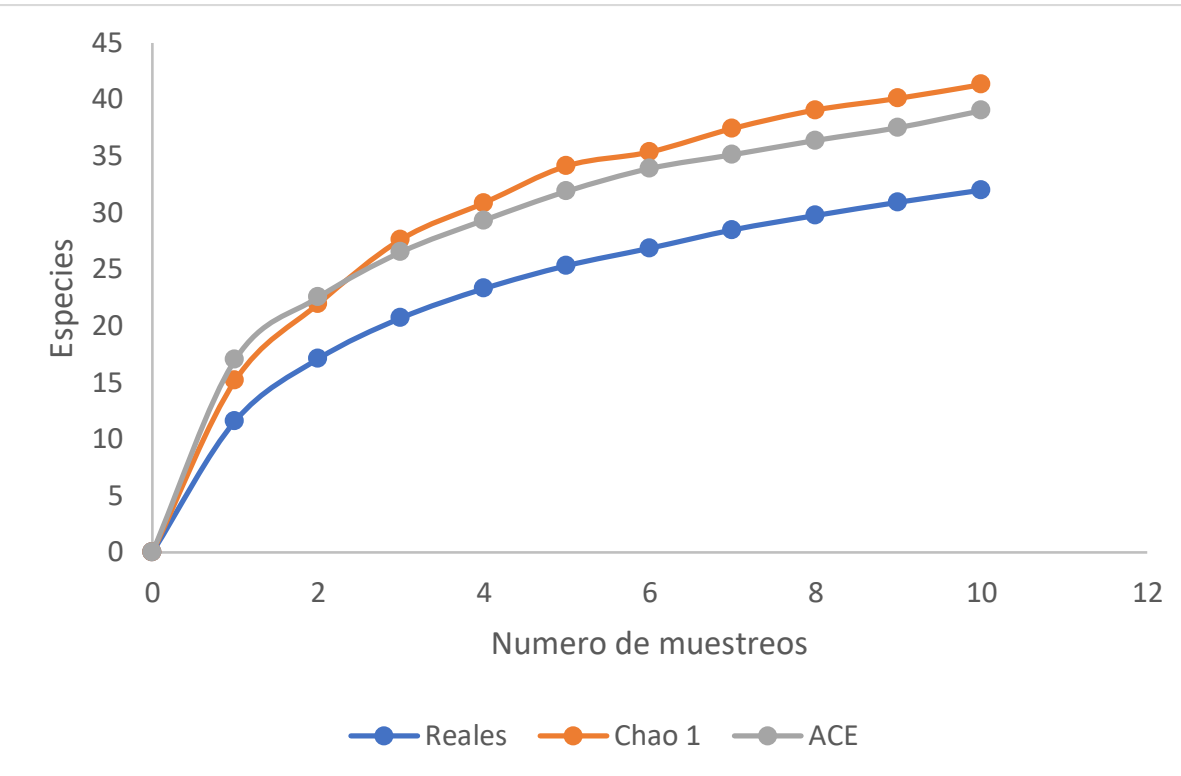

Fig. 5. Curva acumulativa de especies migratorias registradas en El Valle de Antón.

\section{Horas de Actividad y Comportamiento en la zona}

La actividad de los individuos registrados fue mayor en horas de la mañana, entre las 7:00.-11:00. Horas. Alcanzado su hora pico de actividad a las 12:00 horas, con una disminución de manera significativa en las próximas horas; logrando avistar algunos individuos en horas de la tarde entre 16:00.-18:00. horas (Fig. 6). 


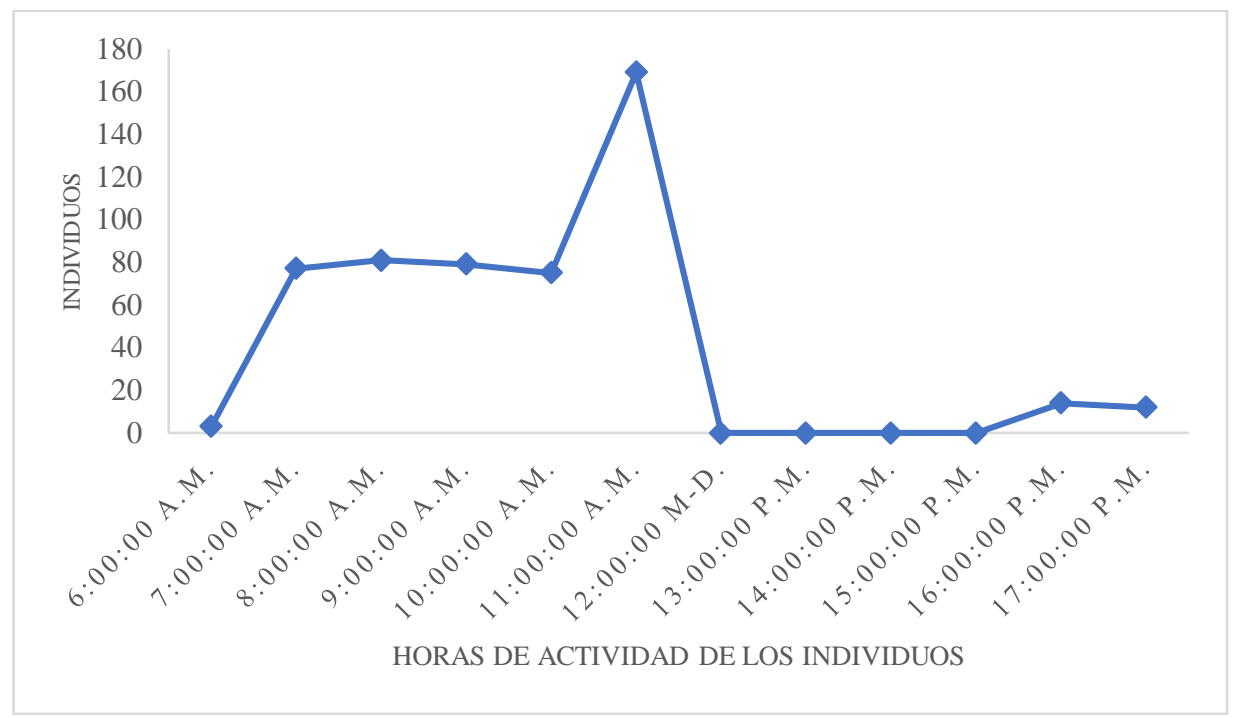

Fig. 6. Actividad por horas de los individuos.

El comportamiento de los individuos registrados se basó en su mayoría, en la acción de vuelo, movimiento o alimentación. En su minoría se encontraban posadas o perchadas sobre ramas de árboles y cableados eléctricos (Fig. 7).

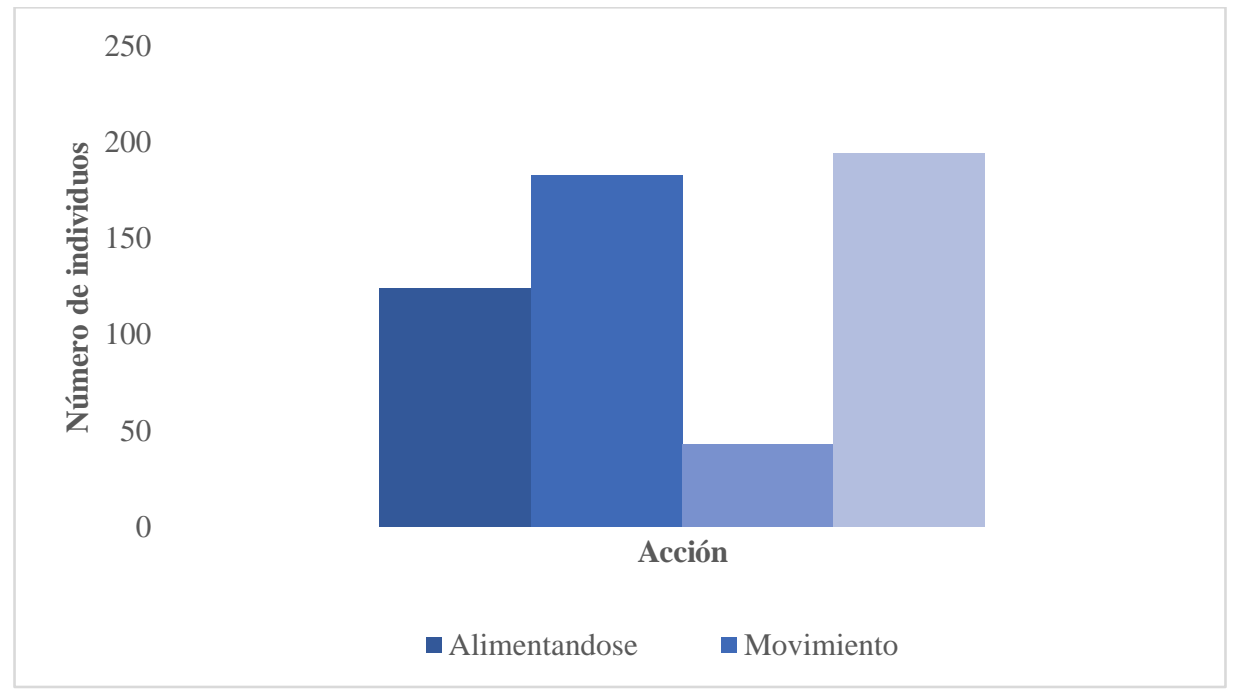

Fig. 7. Comportamiento de los individuos observados en El Valle de Antón. 


\section{Frecuencia de registro/observación}

Las especies Setophaga petechia y Setophaga castanea, presenta la mayor frecuencia de observación (90\%), seguidos de Oreothlypis peregrina y Piranga olivacea (80\%); Vireo flavoviridis y Vireo olivaceus (70\%) (Fig. 8).

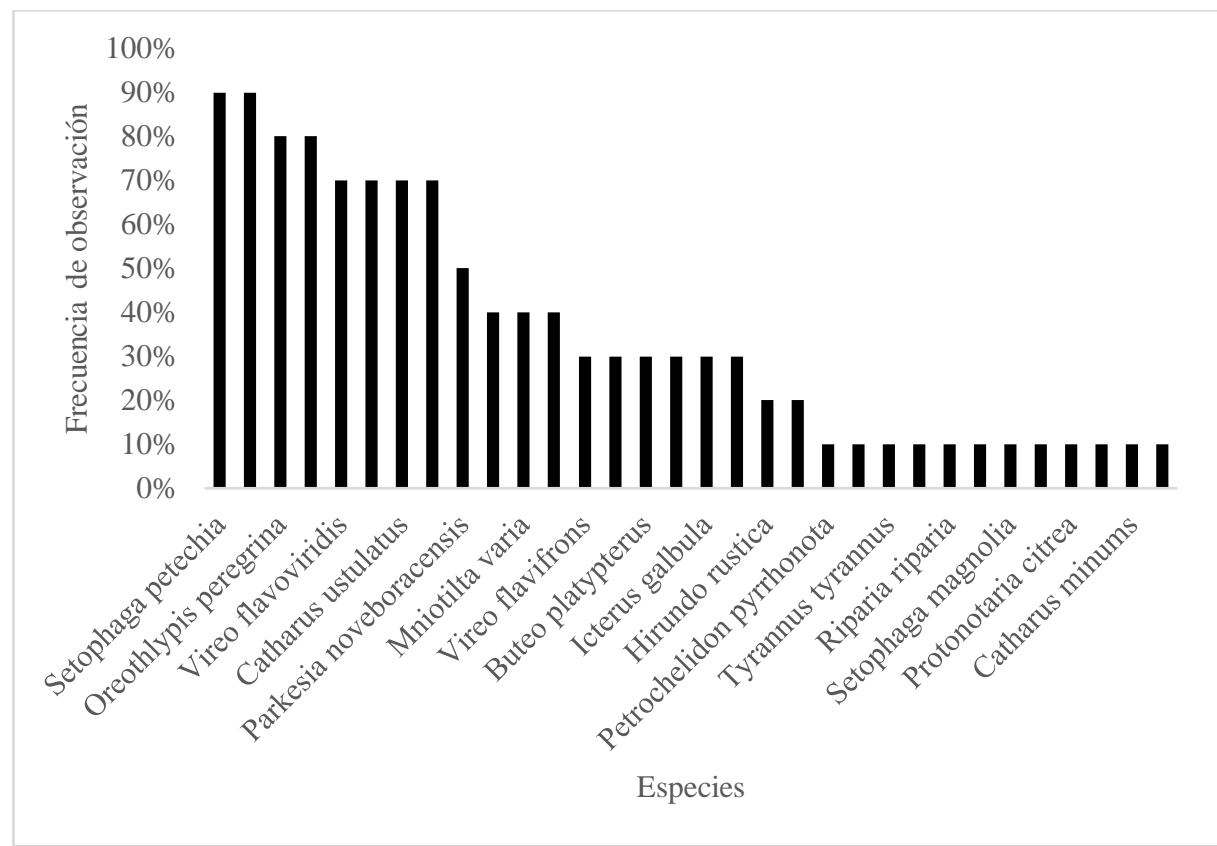

Fig. 8. Frecuencia de observación de las especies, reflejada en porcentaje.

\section{DISCUSIÓN}

\section{Riqueza de especies}

De 150 especies de aves migratorias reportadas para Panamá (Angehr \& Dean, 2010), se registraron 32 especies en El Valle de Antón, lo equivalente al $22 \%$ de estas aves registradas para el país, siendo reportadas entre los meses de octubre y noviembre, meses correspondientes a la migración invernal boreal dada aproximadamente entre los meses de noviembre a abril (Howell \& Webb, 1995). La presencia de especies migratorias en el Valle de Antón se debe a la combinación de dos factores principales: su composición natural/urbana y ubicación dentro del país. En general, la mayoría de las aves migratorias del norte evitan el interior del bosque y prefieren los bordes, 
rastrojos y áreas afectadas por el hombre, como claros y jardines (Ridgely \& Gwynne, 1993) de la cual podemos encontrar zonas o espacio de esta índole en el Valle; la mayoría de las especies realizan vuelos migratorios altitudinales siguiendo cadenas montañosas (Serrano y Garitano, 1996) entre los $500 \mathrm{~m}$ a 2,000 m (Deinlein s.f.), por ende, el Valle de Antón al encontrarse a una altura aproximada de $701 \mathrm{~m}$ a 1,200 m (ANAM, 2010), lo vuelve un lugar auspicio y directo de descanso para los individuos que realizan movimientos a gran altura, y prefieren no descender a tierras bajas, lo que provoca una gasto energético mayor (Arosemena, 2006).

La especie Cathartes aura, posee poblaciones migratorias (grupos migran desde Norteamérica) como residentes en Centroamérica (incluyendo Panamá) (eBird, 2013). Los grupos migratorios pueden ser observados junto a las especies Buteo platypterus y Buteo swaisoni, especies con las cuales comparten fecha y ruta de migración a gran medida, lo cual no es raro de ver a las tres especies juntas durante este periodo (eBird, 2013). Los individuos observados en grupos junto a las especies B.platypterus y B.swainsoni, fueron considerados migratorios para el estudio.

La especie Elanoides forficatus es considerada como no nativa para Panamá, solamente reproductiva, en temporada no reproductiva migra principalmente hacia el Sur, mientras otras bandadas migratorias se dirigen hacia al Norte (Birdlife, 2020). Por lo cual la especie fue considerada migratoria para el estudio.

\section{Esfuerzo}

La curva acumulativa de especies indica que se logró registrar el mayor número representativo de especies migratorias en El Valle de Antón, ya que no hubo un aumento significativo de especies en los últimos muestreos. Según los estimadores no paramétricos Chao 1 y Ace, se obtuvo el $77 \%$ y $82 \%$ respectivamente, dando indicio que en el lugar podemos encontrar aproximadamente hasta 40 especies de aves migratorias. Estas especies al ser animales transitorios de zonas y permanecer pocos días o horas en un área determinada, dificulta su observación en dichos lugares (Deinlein, 1999). 
En comparación a otras zonas similares en cuanto altura e intervención humana (a mayor escala que en El Valle de Antón), se ha reportado un número menor de especies migratorias. Por ejemplo, Araúz y González (2010) estudio en el Cerro Canajagua, Provincia de Los Santos. Reporto 18 especies de aves migratorias, aunque con diferencias en la composición de especies, todo indica que la alta intervención y destrucción de los bosques de Azuero ha incidido negativamente en la biodiversidad del lugar (Araúz y González, 2010).

También se puede mencionar el trabajo realizado por Garcés y Angher (2006). El cual registran 16 especies migratorias para la provincia de Coclé. Sin embargo, su estudio se basó en el registro por el método de redes de niebla (en contraste con nuestro estudio realizado por el método de búsqueda generalizada), posible razón por la cual obtuvieron un menor número de especies. Aun cuando las redes de niebla logran registran una gran variedad de especies poco frecuente de observar, fallan en detectar la mayoría de las aves del dosel, frugívoras de gran tamaño e insectívoras de estrato medio, debido a que la mayoría de estas se alimentan en bandadas mixtas en estratos altos y logran evadir las mallas al oír los llamados de alerta de las demás especies (Polanco et al., 2015). En su mayoría las especies migratorias se alimentan a nivel del dosel (Naranjo et al., 2012).

\section{Horas de actividad y Comportamiento en la zona}

Las horas de actividad de las especies se puede contrastar con su periodo de migración por grupo. La mayoría de las aves cantoras (Orden Passeriformes) migran durante la noche, donde las condiciones son más favorables (temperaturas más frescas y aire calmado) (Deilein, 1999), estimando su tiempo de llegada a una región o zona durante horas de la mañana. Siguiendo patrones comportamentales de forrajeo, movimiento y descanso; evitando horas de sol (Reyes et al., 2015), sus horas de actividad están concentrada durante las primeras horas del día y al finalizar la tarde (en el caso de especies migratorias, horas de inicio para migrar), reduciéndose al llegar al medio día (Franke, 2015). En cuanto a grupos de aves rapaces (Orden Cathartiformes y Accipitriformes) las cuales migran de día utilizando las masas de aire caliente al llegar lo medio día (Deinlein, 1999), desplazando de una zona o lugar al llegar estas horas, lo que reduce su registro de actividad en próximas horas del día en la región. 
En cuanto a su comportamiento, el $80 \%$ de la actividad de las aves es empleado para el forrajeo (Gutiérrez, 1998). En cuanto las aves migratorias, realizan movimientos estacionales durante su periodo de migración en busca de lugares de alimentación, anidación y descanso (Cueto, 2105). Estableciendo estas acciones en su mayoría del tiempo en lugares considerados propicios para estas actividades por las aves.

\section{Frecuencia de registro/observación}

La frecuencia de observación de los individuos en El Valle de Antón, está dominada por miembros del Orden Passeriformes, siendo su mayoría especies de la familia Parulidae, las cuales son aves que se encuentran principalmente en tierras altas, viajando en bandadas en busca de alimento, seguido por especie de la familia Vireonidae, las cuales siguen un patrón de comportamiento similar (Ridgely \& Gwynne 1993), estableciéndose en un lugar determinado por periodos de tiempo donde la alimentación pueda ser constante (Araya y Millie, 2005). En cuanto a los miembros de la familia Hirundinidae, a pesar de poseer un gran número de individuos en su único registro, debido a viajar en grandes grupos migratorios; siguen en su mayoría rutas de migración asociadas a tierras bajas o cuerpos de agua (Wetmore et al., 1984), lo que dificulta su observación en puntos altos del país. El resto de los grupos (Cathartiformes y Accipitriformes) presentaron un porcentaje de observación menor al 50\%. Debido a las grandes extensiones de bosque que aun cubren gran parte del Valle, forman una barrera de árboles que absorben las corrientes de aire caliente, impidiendo que estas llegue a grandes alturas y puedan ser utilizadas por las aves rapaces para comenzar o emplear el vuelo a través de este, dificultando su proceso de migración; razón por la cual estos grupos prefieren grandes cordilleras secas o zonas urbanizadas, donde grandes masas de aire caliente llegan a la atmosfera y pueden ser utilizadas por estas a la hora del vuelo (Márquez et al., 2005).

\section{COCLUSIONES}

El Valle de Antón, por su posición geográfica, altura, composición vegetal y zonas urbanas presentes con jardines, rastrojo y representación floral en el poblado; es un sitio propicio para la observación de aves migratorias neárticas durante su proceso de migración, donde en su mayoría; las especies utilizan el lugar como zona de paso, alimentación 
y en menor proporción para descanso antes de seguir las rutas establecidas.

A pesar del avance urbanístico del Valle, su debido cuidado de jardines $\mathrm{y}$ zonas verdes ha permitido que este cuente con las condiciones predilectas para las aves migratorias, por ende, el cuidado de este lugar podría aumentar la frecuencia anual de observar estas especies dentro de la zona, siendo tomado en cuenta como punto de visita en cada migración anual realizada.

\section{AGRADECIMIENTO}

A Jazmín Alvarado por su apoyo en temas de transporte, brindado el apoyo de un automóvil para el desplazamiento desde la Ciudad de Panamá hasta El Valle de Antón.

Al Grupo Biológico BioMundi por su apoyo en cuanto al financiamiento del proyecto y equipo para la elaboración de este.

\section{REFERENCIAS}

ANAM (Autoridad Nacional del Ambiente). 2010. Atlas Ambiental de la República de Panamá. Primera Versión. Gobierno Nacional de la República de Panamá. 190 pp.

Angehr, G., \& Dean, R. 2010. The Birds of Panama A Field Guide. Zona Tropical Editor in Chief: John K. McCuen. Book design: Zona Creativa S.A., Publication from Comstock Publishing Associates. Division of Cornell University Press. Ithaca and London. 456 pp.

Araúz, J. y González, D. 2010. Aves de Cerro Canajagua, Provincia de Los Santos, Panamá. Universidad de Panamá. Tecnociencia 2010, Vol. 12, No. 2. 138 pp.

Araya, B. y Millie, D. 2005. Guía de Campo de las Aves de Chile. Editorial Universitaria. Novena Edición. 401 pp.

Arosemena, M. 2006. Atlas Visual de la Ciencia. Aves. Editorial Sol 90. $95 \mathrm{pp}$. 
AUDUBON. 2019. Lista de aves de Panamá. Comité de registros, Sociedad Audubon de Panamá. 16 pp.

AUDUBON. 2020. Guía de aves de América del Norte. Recuperado el 10 de mayo de 2020, de AUDUBON https://www.audubon.org/es/guiade-aves.

BirdLife International. 2020. Species factsheet: Elanoides forficatus. Retrieved June 06, 2020, form http://www.birdlife.org.

Cueto, V. 2015. Las Aves Migratorias de América del Sur. Nuevas Técnicas Revelan Información sobre su Comportamiento. Asociación Civil Ciencia Hoy; Ciencia Hoy Vol. 142. Art 7pp.

Deinlein, M. Sin fecha. Conceptos básicos sobre las aves migratorias Neotropicales. Migratory Bird Center of the Smithsonian Nacional Zoological Park. E.E.U.U.

Deinlein, M. Sin fecha. Si se tienen alas, a volar se dijo: las adaptaciones de las aves para la migración. Migratory Bird Center of the Smithsonian Nacional Zoological Park. E.E.U.U

Deinlein, M. 1999. Conceptos básicos sobre las aves migratorias Neotropicales. Smithsonian’s National Zoo \& Conservation Biology Institute.

eBird Centroamérica. 2013. Migración de aves rapaces. Recuperado el 10 de mayo de 2020, de https://ebird.org/camerica/news/migracion-de avesrapaces\#: : :text=Turkey\%20Vulture\%20(Cathartes\%20aura),el\%2 0mundo\%20que\%20Buteo\%20swainsoni.

Eisermann, K. 2006. Evaluation of waterbirds populations and their conservation in Guatemala. Waterbirds Conservation for the Americas. Report. Sociedad Guatemalteca de Ornitología. 74pp.

ETESA (Empresa de Transmisión Eléctrica). 2007. Hidrometeorología Panamá. Mapa de Clasificación Climática (según KÖPPEN). Recuperado el 12 de mayo de 2020, de http://www.hidromet.com.pa/Mapas/Mapa_Clasificacion_Climatica_K OPPEN_2007_Panama.pdf. 
Franke, I. 2015. Patrones de Actividad diaria de las Aves en la Zona Alto Andina. Recuperado el 12 de mayo de 2020, de http://avesecologaymedioambiente.blogspot.com/2017/08/patrones-deactividad-diaria-de-las.html.

Garcés, P. y Angher, G. 2006. Estudio de la diversidad, similitud y dominancia de aves en 10 sitios de la región occidental, provincia de Coclé. Universidad de Panamá, Tecnociencia 2006, Vol. 8, №2.

Google. Sin fecha. [Mapa del Valle de Antón, Panamá en Google earth]. Recuperado el 13 de enero, 2020, de: https://earth.google.com/web/search/El+Valle+de+Ant\%c3\%b3n,+Pan am\%c3\%a1/@8.6200576,80.13582225,700.85138564a,18387.531683 5d,35y,0h,0t,0r/data=CoYBGlwSVgolMHg4ZmFjNThjMGU4YmM2 OTI1OjB4YWEyN2VmYjgzNDgzM2U0Yxk4RleONTghQCHO5zen bQhUwCobRWwgVmFsbGUgZGUgQW50w7NuLCBQYW5hbcOhG AIgASImCiQJGB4alZ12IkARbTo2rdNRIkAZSzXFFs3uU8AhzhiBps fzU8A

Gutiérrez, G. Estrategias de Forrajeo. 1998. The University of Texas at Austin. 41 pp.

Holdridge, L. 1979. Ecología basada en zonas de vida. Traducción de la primera edición. Instituto Interamericano de Ciencias Agrícolas, San José, Costa Rica. 216 pp.

Howell, S.N.G., \& Webb, S. 1995. A Guide to the Birds of México and Northern Central America. Oxford Universidad Press, Oxford, 851 pp.

IGNTG (Instituto Geográfico Nacional Tommy Guardia). 1988. Atlas Nacional de la República de Panamá. Tercera Edición de Panamá. 222 pp.

The Cornell Lab of Ornithology. Bird Guide. Retrieved May 10, 2020, from:

https://www.allaboutbirds.org/news/?_hstc=161696355.adcba91d4f4 043aa20bd92ae7884a933.1588906260652.1589404946821.158941894 $5101.8 \& \_$hssc $=161696355.12 .1589418686247 \& \_h s f p=4282247039$ 
\#/_ga=2.88926939.1920556623.1589404945-

1170823259.1588906240 .

Márquez, C., Bechard, M., Gas,t F. y Vanegas, V.H. 2005. Aves Rapaces Diurnas de Colombia. Instituto de Investigación Alexander Von Humbolt. Bogotá, D.C.-Colombia. 394 pp.

Méndez, E. 1969. Una Breve Introducción a las Aves de Panamá. Instituto Conmemorativo Gorgas de Estudios de la Salud. Universidad de Panamá, Facultad de Ciencias Naturales, Exactas y Tecnología, Escuela de Biología. Edición Privada. 59 pp.

Moreno, C. E. 2001. Métodos para medir la biodiversidad. M\&TManuales y Tesis SEA, vol.1. Zaragoza, 84 pp.

Naranjo, L.G, Amaya J. D, Eusse-González D. y Cifuentes-Sarmientos, Y. 2012. Guía de las especies migratorias de la Biodiversidad de Colombia. Aves. Volumen 1. Ministerio de Ambiente y Desarrollo Sostenible. WWF. Colombia, Bogotá, D.C Colombia. 708 pp.

Polanco, J. M., Ospina, A. D., Arango, D. G., Snaider J. G. y Humberto, M. G. 2015. Efectividad de las redes de niebla para determinarla riqueza de aves en un bosque montano de los Andes Centrales (Salento, Quindío, Colombia). Revista de Investigaciones. Universidad de Quindío. Colombia, 27(1): 75-88; 2015.

Ocampo, N. 2010. El Fenómeno de la Migración en aves: una mirada desde la Orinoquia. Universidad Escuela Politecnica Javeriana del Ecuador. Departamento de Ecología. 13 pp.

Ralph, J.C., Geupel, G.R., Pyle, P., Matin, T.E., Desante D.F. y Milá, B. 1996. Manual de métodos de campo para el monitoreo de aves terrestres. UNited States Department of Agricul. General Technical Report. 45pp.

Reyes, A. R., Jiménez, J. E., \& Rozzi, R. 2015. Daily patterns of activity of passerine birds in a Magellanic sub-Antarctic forest at Omora Park (55S), Cape Horn Biosphere Reserve, Chile. Polar Biol 38:401-411. DOI 10.1007/s00300-014-1596-5 
Ridgely, R., \& Gwynne, J. 1976. A field to the Birds of Panama, with Costa Rica, Nicaragua, and Honduras. Second Edition. Princeton University Press. 519 pp.

Ridgely, R. y Gwynne, J. 1993. Guía de las aves de Panamá, incluyendo Costa Rica, Nicaragua y Honduras. Primera edición en español. Asociación Nacional para la Conservación de la Naturaleza (ANCON). $614 \mathrm{pp}$.

Serrano, V. y Garitano, A. 1996. Biodiversidad de Aves a nivel del paisaje en bosques Andinos y estudios de sus procesos dinámicos. Fundación Cali para la Promoción de la Investigación y la Tecnología.

Wetmore, A., Pasquier, R. F., \& Olson, S. L. 1984. The birds of the Republic of Panama: Part 4. Hirundinidae (Swallows) to Fringillidae (Finches). Smithsonian Institution Press, Washington, D.C., 704 pp. 


\begin{tabular}{|c|c|c|c|}
\hline Orden & Familia & Especies & Nombre común \\
\hline Cathartiformes & Cathartidae & Cathartes aura & $\begin{array}{l}\text { Gallinazo } \\
\text { Cabecirrojo }\end{array}$ \\
\hline \multirow[t]{4}{*}{ Accipitriformes } & Accipitridae & Elanoides forficatus & Elanio Tijereta \\
\hline & & $\begin{array}{l}\text { Ictinia } \\
\text { mississippiensis }\end{array}$ & Elanio Migratorio \\
\hline & & Buteo platypterus & Gavilán Aludo \\
\hline & & Buteo swainsoni & $\begin{array}{l}\text { Gavilán de } \\
\text { Swainson }\end{array}$ \\
\hline \multirow[t]{19}{*}{ Passeriformes } & Tyrannidae & Tyrannus tyrannus & Tirano Norteño \\
\hline & & $\begin{array}{l}\text { Empidonax } \\
\text { virescens }\end{array}$ & $\begin{array}{l}\text { Mosquerito } \\
\text { Verdoso }\end{array}$ \\
\hline & Vireonidae & Vireo olivaceus & Vireo Ojirrojo \\
\hline & & Vireo flavoviridis & $\begin{array}{l}\text { Vireo } \\
\text { Verdiamarillo }\end{array}$ \\
\hline & & Vireo flavifrons & $\begin{array}{l}\text { Vireo } \\
\text { Pechiamarillo }\end{array}$ \\
\hline & Hirundinidae & $\begin{array}{l}\text { Petrochelidon } \\
\text { pyrrhonota }\end{array}$ & $\begin{array}{l}\text { Golondrina } \\
\text { Risquera } \\
\end{array}$ \\
\hline & & Hirundo rustica & $\begin{array}{l}\text { Golondrina } \\
\text { Tijereta }\end{array}$ \\
\hline & & Riparia riparia & $\begin{array}{l}\text { Golondrina } \\
\text { Ribereña }\end{array}$ \\
\hline & Turdidae & Catharus ustulatus & $\begin{array}{l}\text { Zorzal de } \\
\text { Swainson }\end{array}$ \\
\hline & & Catharus minimus & Zorzal Carigrís \\
\hline & Parulidae & Setophaga petechia & Reinita Amarilla \\
\hline & & $\begin{array}{l}\text { Setophaga } \\
\text { pensylvanica }\end{array}$ & $\begin{array}{l}\text { Reinita } \\
\text { Flanquicastaña }\end{array}$ \\
\hline & & Setophaga castanea & $\begin{array}{l}\text { Reinita } \\
\text { Pechicastaña }\end{array}$ \\
\hline & & Setophaga ruticilla & Candelita Norteña \\
\hline & & Setophaga fusca & $\begin{array}{l}\text { Reinita } \\
\text { Gorguinaranja }\end{array}$ \\
\hline & & $\begin{array}{l}\text { Setophaga } \\
\text { magnolia }\end{array}$ & $\begin{array}{l}\text { Reinita } \\
\text { Colifajeada }\end{array}$ \\
\hline & & Mniotilta varia & Reinita Trepadora \\
\hline & & $\begin{array}{l}\text { Oreothlypis } \\
\text { peregrina }\end{array}$ & Reinita Verdilla \\
\hline & & $\begin{array}{l}\text { Geothlypis } \\
\text { Philadelphia }\end{array}$ & Reinita Enlutada \\
\hline
\end{tabular}

Guevara y Samudio 


\begin{tabular}{|l|l|l|l|}
\hline & & $\begin{array}{l}\text { Vermivora } \\
\text { chrysoptera }\end{array}$ & Reinita Alidorada \\
\hline & Protonotaria citrea & $\begin{array}{l}\text { Reinita } \\
\text { Protonotaria }\end{array}$ \\
\hline & & $\begin{array}{l}\text { Parkesia } \\
\text { noveboracensis }\end{array}$ & $\begin{array}{l}\text { Reinita-Acuática } \\
\text { Norteña }\end{array}$ \\
\hline & Parkesia montacilla & $\begin{array}{l}\text { Reinita-Acuática } \\
\text { Piquigrande }\end{array}$ \\
\hline & Cardinalidae & Piranga rubra & Tangara Veranera \\
\hline & & Piranga olivacea & Tangara Escarlata \\
\hline & & $\begin{array}{l}\text { Pheucticus } \\
\text { ludovicianus }\end{array}$ & $\begin{array}{l}\text { Picogrueso } \\
\text { Pechirrosado }\end{array}$ \\
\hline & Icteridae & Icterus galbula & $\begin{array}{l}\text { Bolsero de } \\
\text { Baltimore }\end{array}$ \\
\hline
\end{tabular}

Anexo 1. Aves migratorias observadas en El Valle de Antón, Provincia de Coclé.

Nombres científicos y comunes basados en AUDUBON (2019).

Recibido 18 mayo y aceptado 13 noviembre 2020 